Article

\title{
Medium-Range Probabilistic Forecasts of Wind Power Generation and Ramps in Japan Based on a Hybrid Ensemble
}

\author{
Masamichi Ohba *(D), Shinji Kadokura and Daisuke Nohara \\ Central Research Institute of Electric Power Industry, 1646 Abiko, Chiba 270-1194, Japan; \\ kdkr@criepi.denken.or.jp (S.K.); nohara@criepi.denken.or.jp (D.N.) \\ * Correspondence: oba-m@criepi.denken.or.jp
}

Received: 20 September 2018; Accepted: 27 October 2018; Published: 29 October 2018

check for updates

\begin{abstract}
This study shows the application of self-organizing maps (SOMs) to probabilistic forecasts of wind power generation and ramps in Japan. SOMs are applied to atmospheric variables obtained from the Japanese 55-year atmospheric Reanalysis JRA-55 over the region, thus deriving classified weather patterns (WPs). Probabilistic relationships are established between the synoptic-scale atmospheric variables over East Japan and the generation of regionally integrated wind power in East Japan. Medium-range probabilistic wind power predictions are derived by SOM as analog ensembles based on the WPs of the multi-center ensemble forecasts. As this analog approach handles stochastic uncertainties effectively, probabilistic wind power forecasts are rapidly generated from a very large number of forecast ensembles. The use of a multi-model ensemble provides better results than a one-forecast model. The hybrid ensemble forecasts further improve the probabilistic predictability skill of wind power generation compared with non-hybrid methods. It is expected that long-term wind forecasts will provide better guidance to transmission grid operators. The advantage of this method is that it can include an interpretative analysis of meteorological factors for variations in renewable energy.
\end{abstract}

Keywords: self-organizing maps; weather patterns; synoptic circulation; multi-model ensemble; wind power

\section{Introduction}

Wind energy is receiving increasing attention due to reasons ranging from climate change to its status as the fastest-growing energy source globally. The global production of wind energy has significantly increased in recent decades [1]. Despite this rapid change, wind energy technology is still considered imperfect. The main factor is the fluctuation in wind energy production that can result in significant and rapid changes in power generation over a short period, which is known as the "ramp" phenomenon [2]. As large-scale wind energy ramp events lead to an increase in power grid instability, they must be balanced by other power sources or storage systems, such as pumped-storage hydroelectricity. Forecasting the timing of ramp events can help grid operators avoid unexpected electricity imbalances. Even with the imminent need for ramp forecasts from power grid operators, obtaining highly accurate ramp forecasts remains an important challenge from a practical point of view.

Deterministic forecasting, which is based on numerical weather prediction, is one of the tools that can provide useful information for decision making by grid operators. However, imperfect boundary conditions, initial conditions, and model formulation (e.g., dynamic core, physics) result in nonlinear error propagation during model integration. While the short-range forecasts that are made using high-resolution models are able to represent certain atmospheric processes more accurately and 
provide detailed representation, the accuracy and skill of the deterministic forecasts diminish with time. To quantify forecast uncertainty, more precise knowledge of the plausible future conditions is required for decision making. In recent decades, ensemble forecast techniques have been developed as a tool for probability forecasting to generate a set of plausible future atmospheric conditions [3]. Medium-range (one or two-week) ensemble forecasts are a crucial method for reducing the social impacts of weather events. They provide significantly more time for preparation and decision making than short-range (i.e., a few days) forecasts. It is conceivable that probabilistic medium-range ensemble forecasts can be useful to create weekly system operation plans of electric power, and then increase the capability of renewable energy ramps, adding value to power grid management by providing more confidence (and less uncertainty) than deterministic forecasts.

In addition to ensemble methods, statistical post-processing is also necessary to calibrate model output. Statistical/empirical post-processing techniques for numerical weather forecasts are frequently used, powerful approaches that improve the impacts of model error or initial boundary conditions. These techniques are now used in various end-user applications, including estimates of renewable energy production. A promising post-processing technique is the analog approach, in which, based on the assumption that if forecasted weather conditions are similar to historical patterns (e.g., the spatial time series of wind speed and direction), then the local weather can be determined. Several studies have explored the use of analog-based methods for producing both deterministic and probabilistic weather predictions [4-9]. Delle Monache et al. [9] showed that the analog approach is useful only to calibrate raw numerical forecasts and generate probabilistic information from a purely deterministic forecast. Some studies have discussed the application of an analog ensemble (AnEn) to regional renewable energy forecasting $[10,11]$.

As a very large amount of forecast data is provided in medium-range ensemble forecasts, efficient tools are required to extract useful information. Self-organizing maps (SOMs), which was developed by Kohonen [12], is one of many data mining techniques that are capable of projecting high-dimensional nonlinear features onto a visually comprehensible two-dimensional map. Attempting to overcome the problem of downscaling a large number of ensemble forecasts, recent studies [7,13-15] have proposed the use of a SOM-based analog technique to estimate the local weather conditions from the ensemble forecasts of the synoptic environment. However, no study has addressed the specific application of using SOMs for medium-range wind power forecasts.

The goal of this study is to evaluate the ability of multi-model ensemble forecasts, in combination with SOM-based analog methods, to forecast probabilities of area-integrated wind power and ramps for medium-range lead times. SOMs were used to identify weather patterns (WPs) over East Japan, while using the analog approach for wind power forecasts up to one week in advance. This method could be categorized as a hybrid ensemble method, as suggested by Eckel and Delle Monache [16], that is skillful compared with that based on a single deterministic forecast. This hybrid ensemble forecast (combined application of a multi-model ensemble and analog ensemble post-processing) offers relatively good prediction skill for wind power generation and its climatological/meteorological interpretation. It is implied that the application of fast techniques will increase decision-making capabilities in the user community, such as electric power transmission system operators. This study is organized as follows. Section 2 provides a description of the dataset and the methods used in this study. Section 3 shows the results of the probabilistic forecast of wind power, while examining the effects of the multi-model ensemble in improving renewable energy predictability skill. Finally, Section 4 provides a summary of the conclusions of this study.

\section{Data and Method}

\subsection{Data}

Three-hourly instantaneous values of atmospheric data for the period 1977-2010 were obtained from the Japanese 55-year Reanalysis (JRA-55) [17], which were downloaded from the JMA Data 
Distribution System (for details http:/ /jra.kishou.go.jp/JRA-55 and for verification [18]). We used sea level pressure (SLP) and surface wind at $10 \mathrm{~m}$. These atmospheric variables were available at a horizontal resolution of approximately $60 \mathrm{~km} / 0.5^{\circ}$. This study focuses solely on integrated wind power generation in the Tohoku region (blue region in Figure 1), where the production of wind-generated power is the highest in Japan. However, the time window of wind power observation is limited to only two years (FY2011/2012, i.e., April 2011 to March 2013). In this study, we used the reconstructed wind power supply data from Ohba et al. [19], from 1977 to 2010, for training our post-process model. This wind power data was historically reconstructed using long-term observational data obtained from weather stations in Japan, called the Automated Meteorological Data Acquisition System (AMeDAS). In this study, a wind power variation that produces $>30 \%$ change in wind power generation in $\leq 6 \mathrm{~h}$ is defined as a "wind ramp event", which is similar to previous study [19]. The positive and negative quick change each qualify as a "ramp-up and ramp-down", respectively.

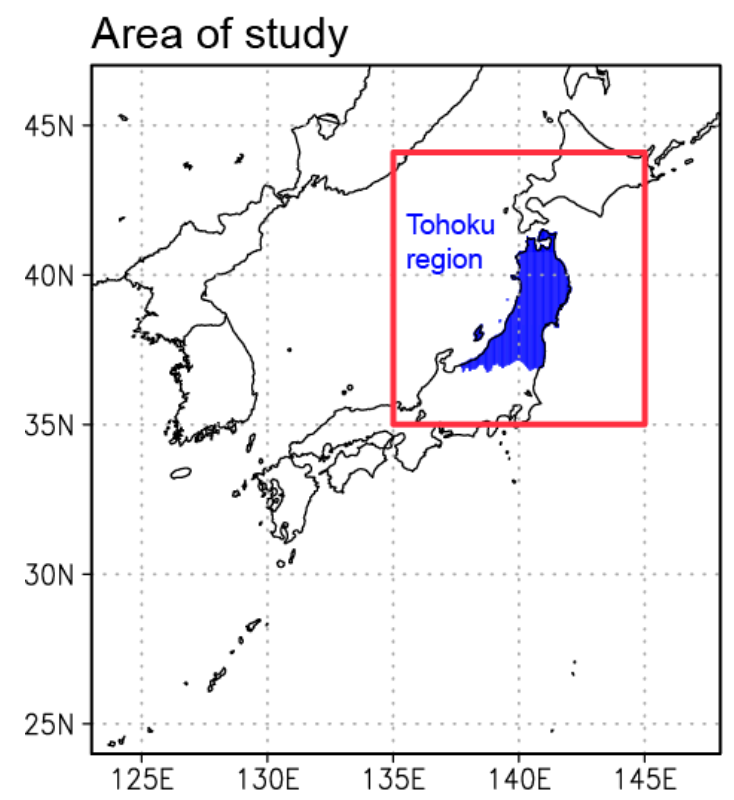

Figure 1. Area of study in East Japan (red solid box) used to define the atmospheric patterns. The blue shading represents the Tohoku region (served by Tohoku Electric Power Co., Inc.), Sendai, Japan.

\subsection{Ensemble Forecasts}

We also used past operational medium-range ensemble forecasts from five weather prediction centers: JMA (Japan Meteorological Agency), NCEP (National Centers for Environmental Prediction), UKMO (United Kingdom Met Office), CMC (Canadian Meteorological Centre), and ECMWF (European Centre for Medium-Range Weather Forecasts). The ensemble forecast data for six-hourly values of sea level pressure and surface wind at $10 \mathrm{~m}$ are obtained from the TIGGE (THORPEX Interactive Grand Global Ensemble) portal at ECMWF from April 2011 to March 2013. As part of the THORPEX (The Observing System Research and Predictability Experiment) research program, this dataset is currently available for non-commercial research purposes only at a two-day delay. The forecast length used was $216 \mathrm{~h}$, while the total ensemble size was 168 . Only the ensemble forecasts initialized at 12:00 UTC were used here to compare the products while creating a multi-model (i.e., multi-forecast center) ensemble based on the data from the five weather prediction centers.

\subsection{SOM Technique}

To establish links between various WPs and their impacts on regionally integrated wind power, an artificial neural network was used in this study. The SOMs technique, which was developed by Kohonen [12], is one of the most commonly used nonlinear pattern recognition techniques. 
The SOMs technique projects high-dimensional data to a visually comprehensible, two-dimensional map. Since SOMs provides a spatially organized set of patterns of data variability, this technique has already been used in many synoptic climatological analyses (readers can refer to Ohba et al. [20]). Patterns are topologically ordered across the SOM array based on pattern similarity such that the farthest point of the array contains the patterns with the largest dissimilarity. As described in previous studies $[7,19,21]$, SOMs offers many advantages for the analysis of WPs.

In this study, we applied SOMs to the atmospheric variables derived from JRA55 around the Tohoku region $\left(135^{\circ} \mathrm{E}-145^{\circ} \mathrm{E}, 35^{\circ} \mathrm{N}-44^{\circ} \mathrm{N}\right)$. The SOM projects these input vectors onto regularly arranged two-dimensional arrays. Each of the arrays, which are referred to as nodes, has one reference vector. For example, a $50 \times 50$ grid SOM comprises 2500 reference vectors, which project onto a map composed of 2500 nodes. The reference vector represents a generalized pattern of input vectors. For more details, refer to other recent study [15]. To train the SOM, we used SLP, surface wind vector, and surface wind speed that showed high correlation to the wind power generation time series. We used the torus-type SOM, instead of the conventional SOM, as it has no edges in the map [22]. While torus SOM increases the computational cost, the learning on neurons is uniformly performed from each direction, since it does not have boundary neurons that result in the improvement of pattern classification from the conventional SOM [22].

The SOM was applied on a three-month basis for the period 1977-2010, i.e., boreal spring (March-April-May: MAM), summer (June-July-August: JJA), fall (September-October-November: SON), and winter (December-January-February: DJF). We mainly present the winter results, during which high wind power generation was observed in Japan [19].

\subsection{SOM-Based Analog Ensemble}

A SOM is used in this study to estimate the wind energy variation in the region by first creating a relationship between atmospheric fields and local wind power generation. Each node in a SOM defines the wind power generation corresponding to each analog WP. Based on this link between the SOM-obtained WPs (represented by reference vectors) and the corresponding regionally integrated wind energy, we obtained a forecast PDF (probability density function) of wind power based on the atmospheric variables of the TIGGE multi-center ensemble. This can be regarded as an alternative to the conventional analog $[4,5]$ or analog-ensemble [9] techniques that have been presented in previous studies. While the original analog-ensemble compares past forecasts with past forecasts, this method compares past forecasts with past analysis. The SOM establishes a nonparametric relationship between the predictor (WPs) and the predictands (wind power), subsequently requiring some statistical assumptions. In this study, the forecast PDF is estimated using a set of past historical power generation data corresponding to the best analogs (atmospheric reanalysis) for the current multi-model ensemble forecast. The observational data for each analog is a member of the analog ensemble [9]. One advantage may be to significantly lower the computational expense by compressing the analogs using SOMs. While the original analog ensemble method $[9,23]$ generally uses a fixed number of analogs (such as 25 in Vanvyve et al. [24]), the number of analogs (i.e., the number of best match) here are determined by the SOM, leading to a difference among the SOM nodes in this method. To capture the spatial time evolution of weather patterns, $\mathrm{a} \pm 3 \mathrm{~h}$ time WP (i.e., $6 \mathrm{~h}$ time window for the analog trend) is also included in the input vector, with all three WPs equally weighted. The predictor variables are also treated equally.

To match the forecast data to the SOM, the same variables are extracted from the TIGGE ensemble data for a particular region. Based on their distance from the reference vectors, each WP of the ensemble forecasts is assigned to its best-match node. Here, 168 forecast patterns are available at six-hour intervals. The $\pm 3 \mathrm{~h}$ WPs for analog trends are obtained by linear interpolation. Finally, the predicted composited PDF is obtained from the PDF, assigning the regional wind power to each node. The Euclid distance between JRA-55 and the ensemble forecasts are used to detect the best-matched node. The atmospheric values at the grid points in the reanalysis were interpolated to model grids 
to yield the corresponding grid maps. We used relatively large-size SOMs compared to previous studies to provide greater detail in the atmospheric patterns that are relevant to wind power variability. A schematic diagram of the algorithm of the downscaling technique is shown in Figure 2, and summarized below.

(1) Nine SOMs are applied to the atmospheric variables (top-left panel). $50 \times 50,80 \times 80$, and $100 \times 100$ SOMs are used. Each SOM is trained separately with absolute wind speed, wind vector, and SLP, i.e., a total of nine SOMs was used (as shown in Table 1).

(2) PDFs of wind power generation and ramp probability are estimated (obtained from observational data; bottom-left panel) for each node of nine SOM in Table 1. To develop a PDF for each SOM node, in addition to the targeted node, samplings are also obtained from eight neighboring nodes that are assigned lower (half) weights compared with the center node.

(3) Using the SOMs obtained in (1), the node that best matches the output of the multi-model ensemble forecasts (top-right panel) is selected from the SOM maps, respectively.

(4) Wind power PDFs are derived by compositing the individual results of ensemble forecasts obtained in (3) (bottom-right panel).

(5) The ensemble composited PDF of wind power generation for the targeted region is obtained from (4).

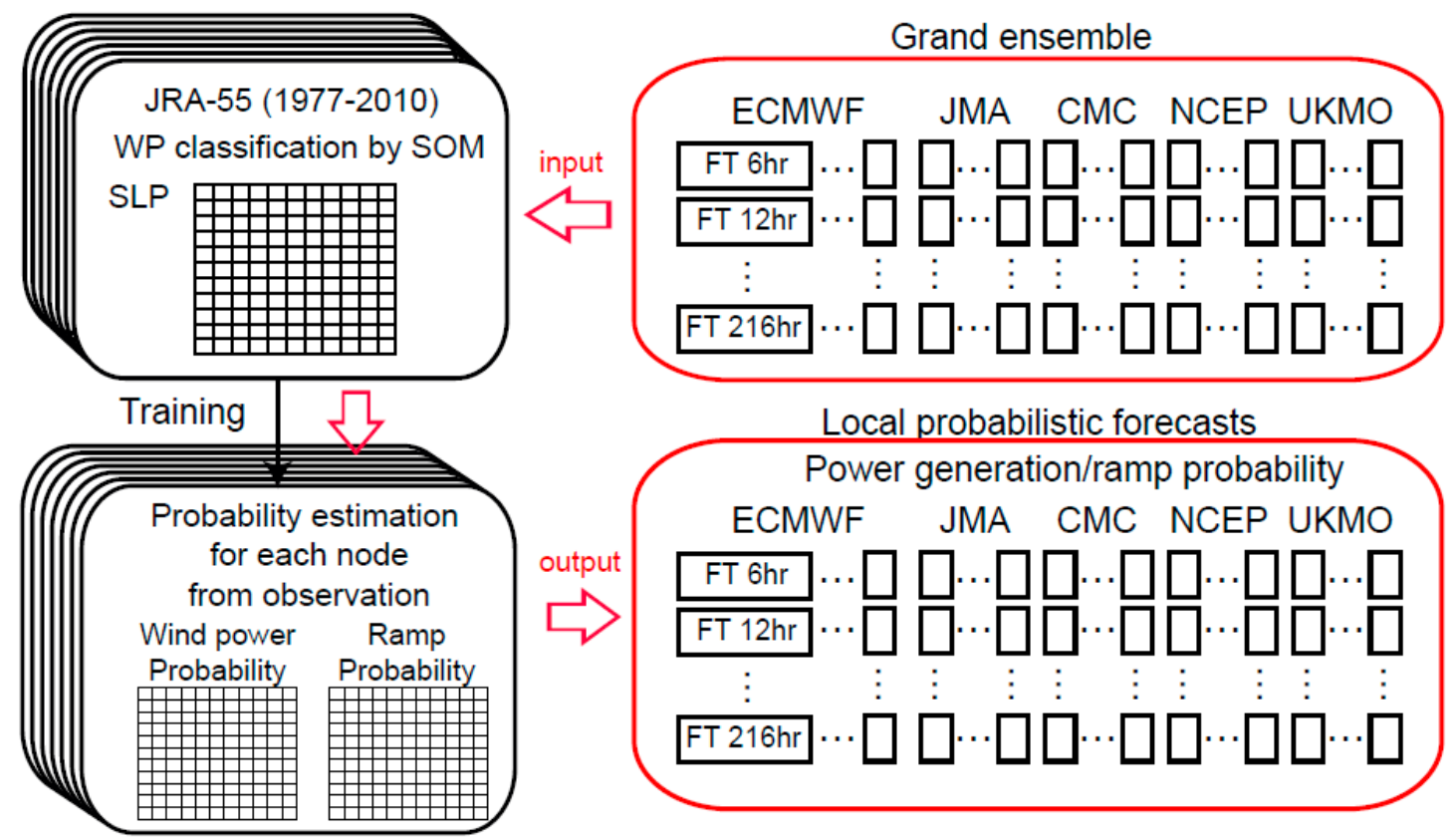

Figure 2. Schematic of the hybrid ensemble using the self-organizing maps (SOMs) technique. The multiple SOM classifications of weather patterns (WPs) are based on the three atmospheric variables during 1977-2011 (top, left). Based on the SOM lattices, the PDF (probability density function) of wind power generation and ramp probability are estimated for each node (bottom, left). By using the SOM lattice, the forecasted wind power generation and variation are obtained (bottom, right) from the 168 members of the multi-center ensemble forecasts (top, right).

The use of a $50 \times 50 \mathrm{SOM}$ results in a mean number of analogs of approximately 10, but the $80 \times 80$ yielded about four, and the $100 \times 100$ yielded about 2.5. We decided to use three different SOM sizes, since the number of analogs is an important parameter of the analog ensemble. This method could be regarded as a SOM-based hybrid multi-model analog ensemble [15]. This analog ensemble is sensitive to the selection of parameters, such as SOM dimension size and atmospheric variables. Sensitivity to the choice of input was tested using other variables, and we determined the combination of parameters that was used in this study. For example, previous studies $[23,24]$ on wind power generation suggested 
that wind speed and direction are important for wind power forecasts. The selection of the variables in this study is consistent with the results of previous studies.

Table 1. Parameters used in the nine SOMs.

\begin{tabular}{|c|c|c|}
\hline \multicolumn{2}{|c|}{ Atmospheric Variables } & \multirow{2}{*}{$\begin{array}{c}\text { SOM Size } \\
50 \times 50\end{array}$} \\
\hline 1. & Sea Level Pressure & \\
\hline 2. & Sea Level Pressure & $80 \times 80$ \\
\hline 3. & Sea Level Pressure & $100 \times 100$ \\
\hline 4. & Sfc. wind vector & $50 \times 50$ \\
\hline 5. & Sfc. wind vector & $80 \times 80$ \\
\hline 6. & Sfc. wind vector & $100 \times 100$ \\
\hline 7. & Absolute wind speed & $50 \times 50$ \\
\hline 8. & Absolute wind speed & $80 \times 80$ \\
\hline 9. & Absolute wind speed & $100 \times 100$ \\
\hline
\end{tabular}

\section{Wind Ramp Prediction Based on Multi-Model Ensemble Forecast}

\subsection{Estimated Wind Power and Ramp}

Generally, synoptic WPs, in relation to large-scale atmospheric conditions, are important for comprehending wind power variations, since they affect near-surface wind [25-28]. Therefore, they can be good predictors [29-34]. For example, a frontal system passage, a low-level jet, and a planetary boundary layer growth can be major factors in wind power variations [35]. Wind power ramps in Japan are mainly caused by large fluctuations in wind speed in relation to the time evolution of synoptic circulation over East Asia [19] that always affects the load generation balance.

Since regional wind power variations can have various atmospheric origins, in addition to being nonlinearly related to various meteorological factors, the classification of synoptic-scale weather background conditions could be useful not only for understanding weather factors, but also for improving wind power forecasts. First, in this section, we present the results of a SOM-based WP classification. Three examples of WPs are presented in Figure 3. The mean atmospheric condition corresponds to the reference vector derived from the $50 \times 50$ SOM analysis for SLP during DJF (i.e., the $3 \mathrm{~h}$ by $3 \mathrm{~h}$ WPs, classified into 2500 nodes). The SOM analysis uses the $24,480{\text { ( } 8 \mathrm{day}^{-1} \times}^{-1}$ 90 days in DJF $\times 34$ years) data as the input vector. The SLP values that are used here are obtained by removing the regional mean SLP values from the original data at each time step. Red and blue shading indicate relatively high and low SLPs, respectively. We have also showed the corresponding wind power generation, ramp-up and ramp-down rates, and the node-mean maximum increase and decrease in wind power generation over a period $\leq 6 \mathrm{~h}$ corresponding with each WP. The patterns are selected as representative WPs that can lead to relatively strong wind power generation or a ramp, which is consistent with the dominant wind power variation patterns in Japan, as has been discussed in previous study [19].

During the winter, East Asian winter monsoons dominantly affect Japan's climate and local weather conditions, which are characterized by cold air outbreaks originating from the negative zonal pressure gradients between the Aleutian Low and the Siberian High [36]. Two typical cyclone tracks are observed around Japan in winter: the southern coastal cyclone track and the Japan Sea cyclone track. In Figure 3, we see the impact of WPs on the regional wind power generation. Corresponding to the distribution of SLP, the regional wind power generation responses to the WPs are significantly different. For example, the WP at the top of Figure 3a, in eastern Japan, is covered by a strong zonal SLP gradient. The SLP gradient is very effective at stably producing wind power over the region, with variations $\leq 6 \mathrm{~h}$ being significantly weaker. However, low-pressure systems approaching the region from the northwest can cause more frequent ramp-ups (Figure 3b). A meridional SLP gradient covers the Tohoku region, which is associated with the low-pressure system located at the north of the region (Figure $3 b$ ), and can result in a rapid increase in surface wind speeds within several hours. 
The cold front in relation to the mid-latitude cyclone passes is clearly seen. In contrast, the SLP gradient over the region that is shown in Figure $3 c$ decreases, due to a reduction of high SLP over southwest Japan, resulting in more frequent ramp-downs. These results imply that different WPs can result in differences in wind power generation and its subsequent stability.

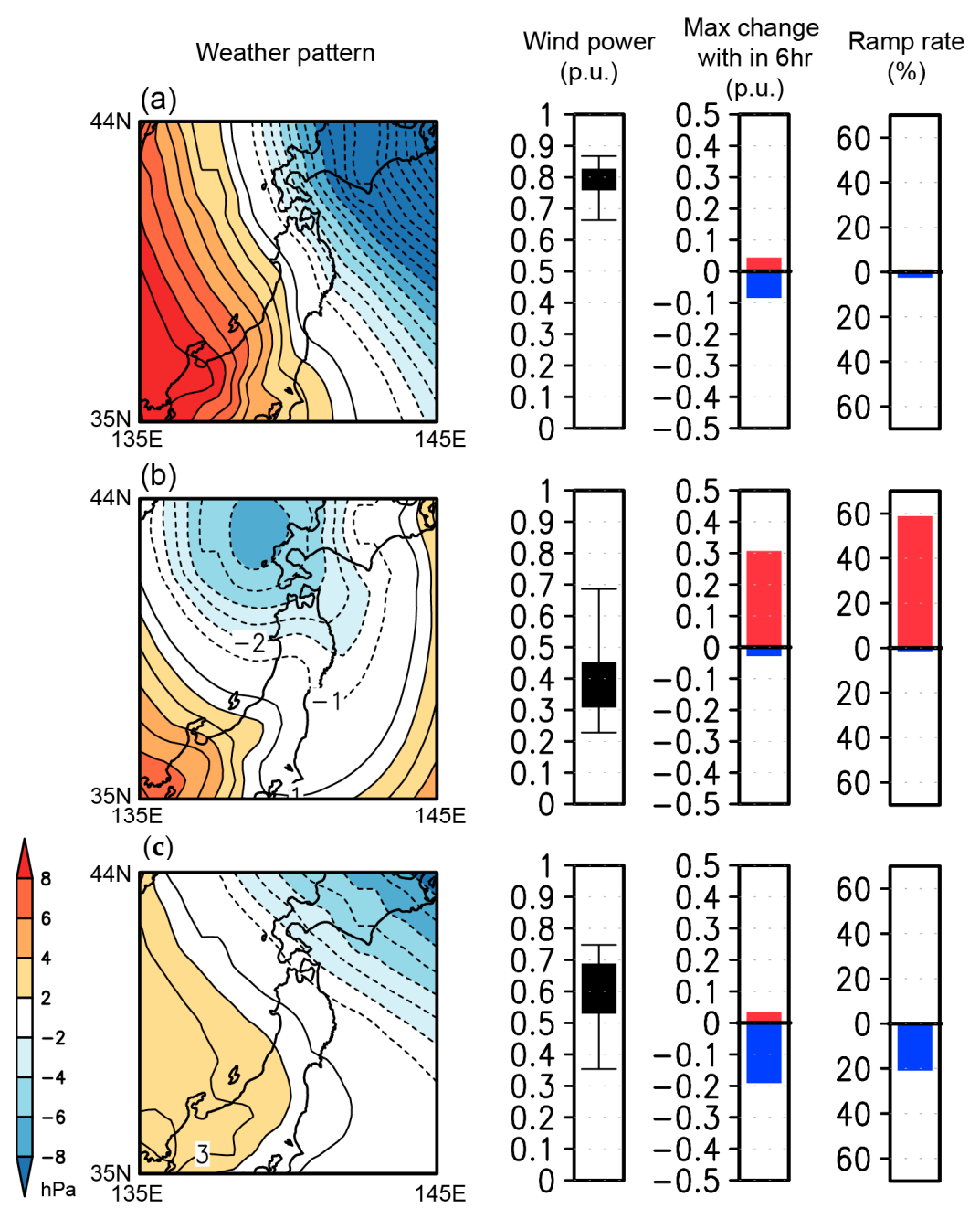

Figure 3. (a-c) Three examples of WPs (SLP) derived from the $50 \times 50$ SOM nonlinear classification (hPa: red and blue shading). Right panels show the WP-related wind power generation (p.u. black error bar for fifth, 25th, 75th, and 95th percentiles), frequency occurrence rate (\%) of ramp-up (red) and ramp-down (blue), and maximum up (red) and down (blue) of wind power generation (p.u.) over $\leq 6 \mathrm{~h}$ for each SOM node.

An example of the relationships between WPs and wind power variations (node mean) on the SOM lattice is presented in Figure 4a. In this figure, a relatively strong wind power generation (i.e., exceeding 0.7-0.8 p.u.) is observed in the bottom-right on the SOM. We find a strong contrast between nodes. This implies that integrated wind power generation in this region is strongly dependent on synoptic WPs. The SOM analysis of wind power variability over a 6-h time zone also allows us to estimate average wind power variations. The occurrence rates of ramp-up and ramp-down events are also shown in Figure 4a. It is clear that the nodes denoting a higher occurrence rate of ramp events also have a coherent spatial pattern in the SOM, implying that both, wind power generation and ramps are highly dependent on synoptic WPs. As denoted by Ohba et al. [19], many nodes share the probability of a ramp-up/down event. 
(a) Relationships between WPs and wind power variations on SOM
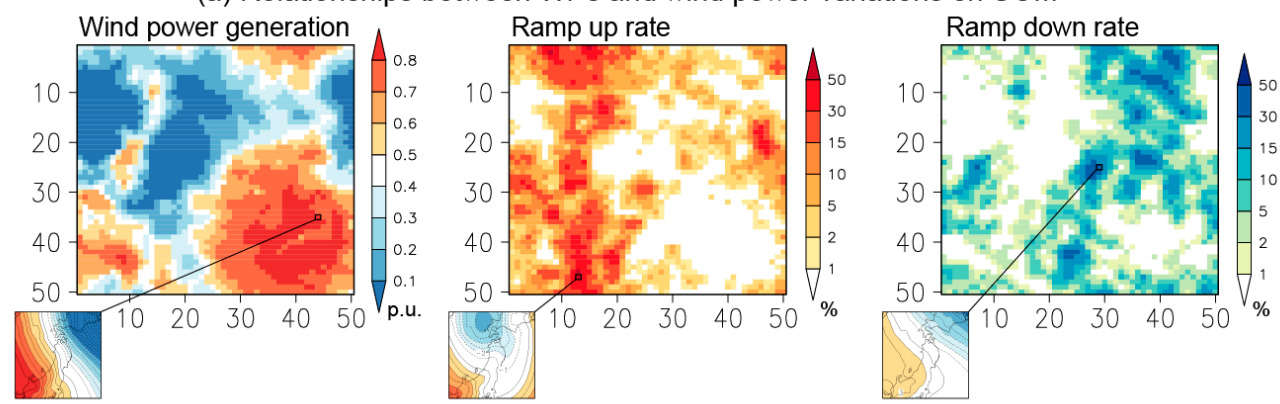

(b) Footprints of multi center grand ensembles on SOM
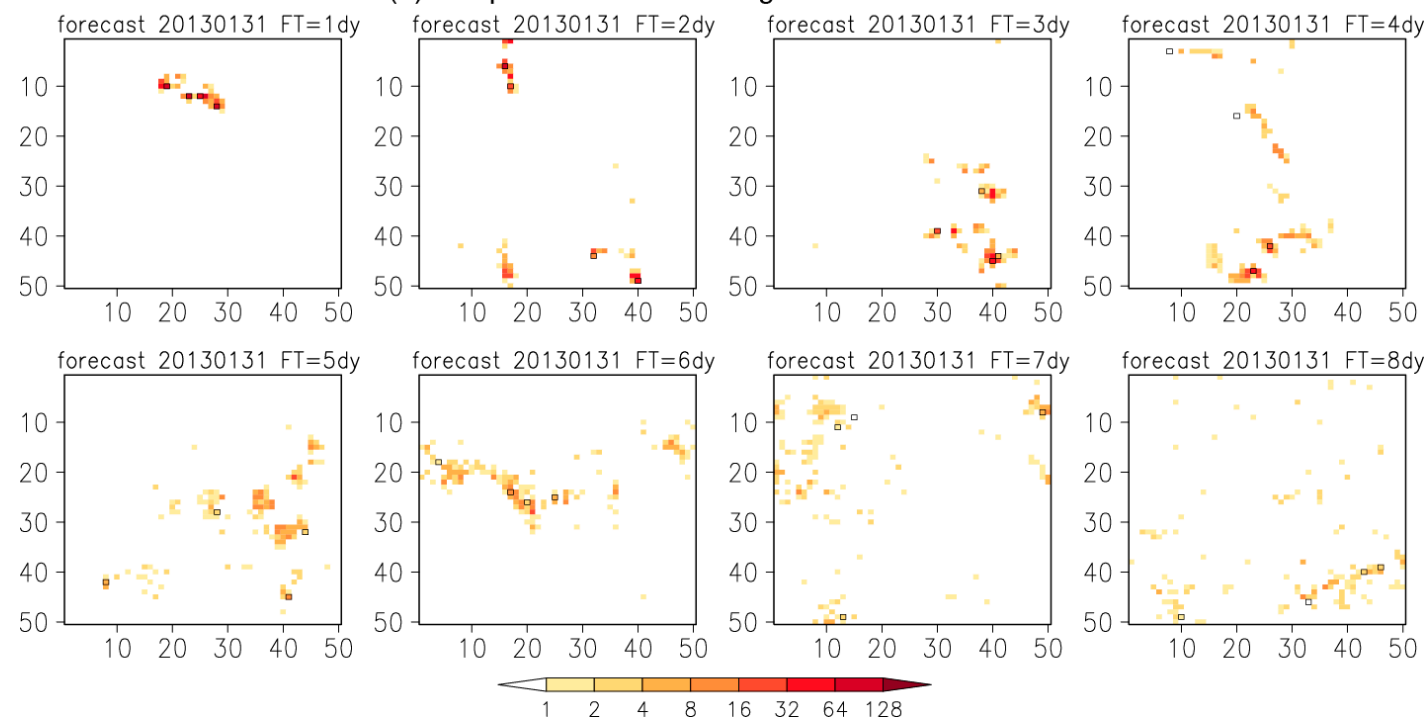

Figure 4. (a) The median values of wind power generation (left) and the frequency occurrence rate (\%) of ramp-up (middle) and ramp-down (right) within each SOM node. Each bottom-left figure shows the correspondence with Figure 3a-c, respectively. (b) The SOM frequency (best-matched) of the 168 multi-center ensemble members on the $50 \times 50$ SOM lattice for forecast days $1-8$ initiated from 1 January 2013. Solid black box represents the actual state.

Figure 5 shows four examples of probabilistic forecasts of wind power generation (top) and ramp (bottom) that are obtained from the multi-model ensembles (MME) of TIGGE through SOM analysis. Gray lines represent the ensemble spread of the 50th percentile output, which was obtained from the multi-model ensembles, while the green shading represents the PDF of wind power generation obtained from the hybrid (multi-model analog) ensemble method. In these cases, throughout the period, the predicted wind power generation is in close agreement with the observational result. The PDF covers the observed wind power generation well, while occasionally overestimating/underestimating the power generation. As for the second half of the period, the predicted wind power generation generally captures the high risk of ramp events relatively well. As the best estimates (50th percentile) of each ensemble member gradually extend, they approach the climatological PDF with reductions in the day-to-day difference.

Probabilistic ramp forecasts are represented by the red (ramp-up) and blue (ramp-down) dots and bars in Figure 5. The ramp probability obtained from the SOM node is represented by a bar, which is the mean of the estimated value of 168 ensembles. The error bar indicates the maximum and minimum values in the nine SOMs. We additionally estimated the ramp probability using the 50th percentile value of each ensemble (represented by the gray line in Figure 5), which is denoted by a dot. The defined ramp-up and down events that are seen in the observed wind power generation (i.e., actual ramp events) are represented by red and blue lines. In these cases, ramp events are forecasted some number of days before the occurrence. 

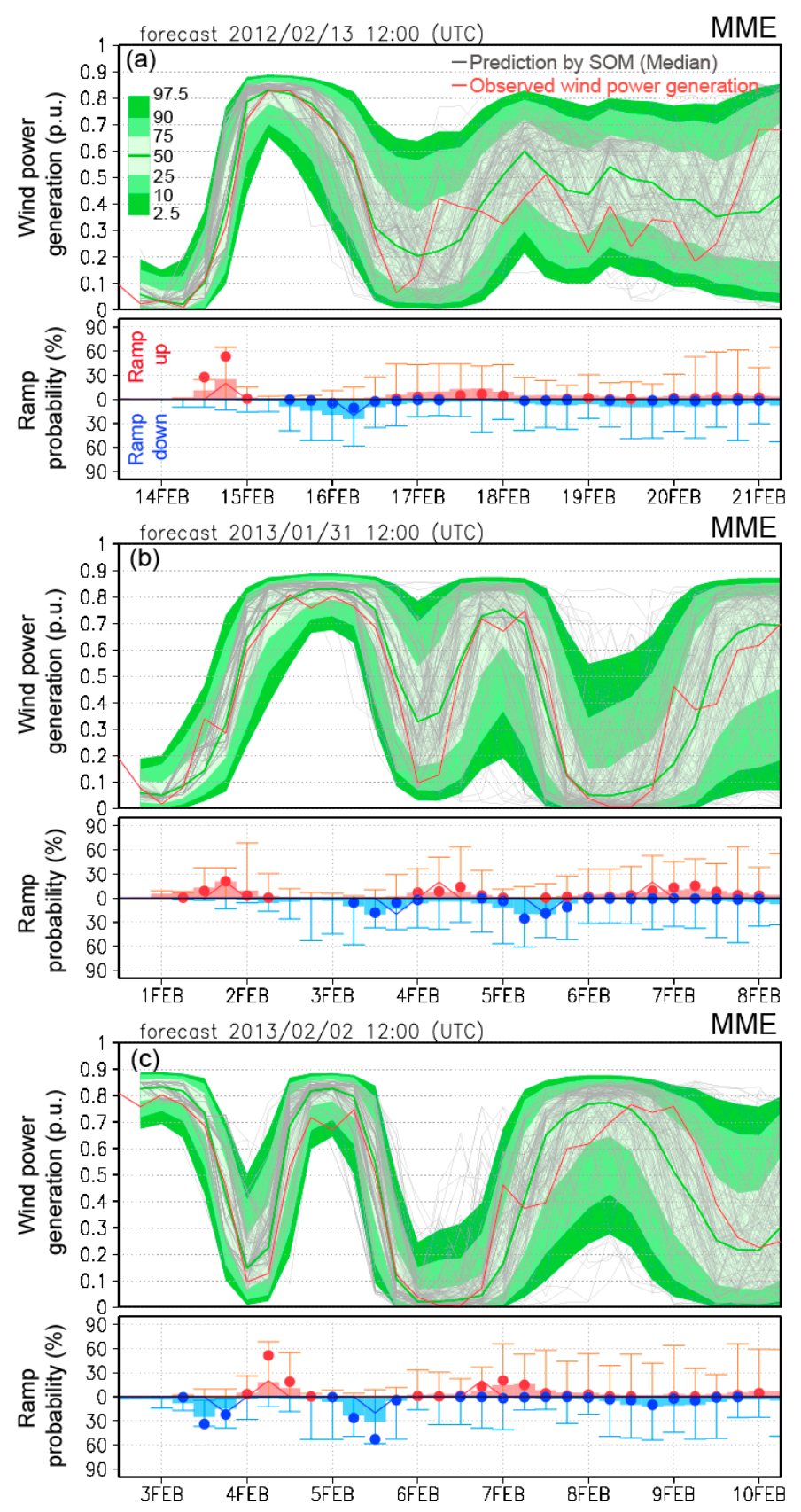

Figure 5. Forecasted 6-h wind power forecasts obtained from the single center ensembles with SOM-based analog-ensemble, initiated from (a) 13 February 2012, (b) 1 January 2013, and (c) 2 February 2013, at 12:00 UTC, as examples of the forecast results. The red line represents the observed wind power generation. PDFs of wind power generation obtained from the hybrid ensemble are represented by green shading. Gray line represents the median values of each ensemble member (total 168 lines). Each figure at the bottom shows the forecasted ramp probability obtained from the SOM nodes, and the medians of each ensemble are represented by red and blue bars and dots, respectively, with the error bar. Observed ramp-up and ramp-down events (actual state) are represented by the red and blue lines, respectively.

Figure $4 \mathrm{~b}$ shows the footprints of the multi-model ensembles on the SOM lattice (Figure $4 \mathrm{a}$ ), which were predicted from 31 January 2013 (corresponding to Figure 5b). The black line box in Figure $4 \mathrm{~b}$ shows the actual WP seen in the SOM (i.e., the best-matching node of the reanalysis). The frequency of occurrence of each SOM pattern results from mapping 168 ensembles $\times$ four times (in one day) to the SOM. If the forecasts are "perfect", a very dark square is identical to the solid 
black box, indicating that all of the forecast ensembles matched the observations. The spread of the colored boxes indicates the range of skill of the ensemble members, which varies by forecast days. The frequencies of WP in the multi-model (168) ensemble extend gradually on the SOM while forming the groups. In this case, the multi-model ensemble forecast captures the atmospheric conditions of the actual state relatively well for forecast day eight (except for forecast day four, which could be related to an underestimation of the rapid decrease in wind power in Figure 5b). The expanse of forecasts on the SOM can be an effective way to visually grasp the broadening of ensembles and the reliability of prediction.

As an example of the intermodel difference, we also show the results of the individual forecast centers separately in Figure 6. We find a relatively large diversity of wind power forecasts among the models. In this case, the UKMO model captures the ramp-up and ramp-down that occurred on 4 February well. However, two days later, most of the models capture both the ramp-up (on 4 February) and down (on 5 February) well, as seen in Figure 5c. In this case, the ensemble spread is relatively small in the UKMO and NCEP models.
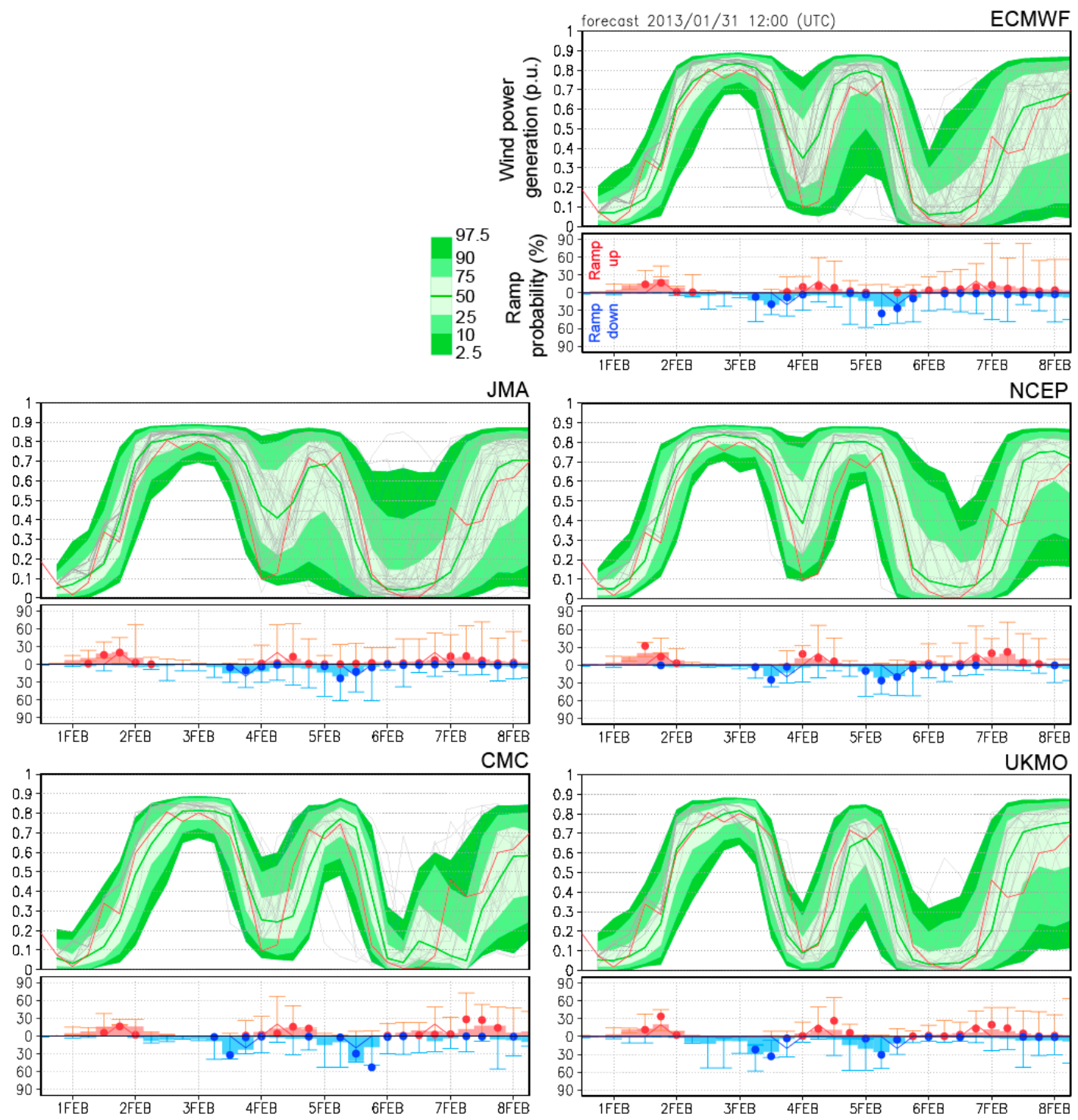

Figure 6. Same as Figure 5, except it is obtained from the five single-center ensembles with SOM-based analog-ensemble initiated from 1 January 2013, at 12:00 UTC. 


\subsection{Forecast Skill of Wind Power Variations}

This subsection evaluates the predictability of wind power generations derived from this method, based on the ensemble forecasts for FY2011/2012. Figure 7 shows the forecasted PDF of wind power generation and ramp probability, obtained from the hybrid multi-model analog ensemble during mid-December to January of 2012/2013. The forecasted PDF for each forecast day (two, three, five, and seven-day lead times) is shown separately. The green shading shows the obtained PDF of the wind power generation, while the dot at the bottom indicates the ramp probability. Moreover, the ramp probability obtained from the ensemble means of SOM nodes are represented by the dots and bars. The results obtained from this method show a relatively wide-range PDF. In the Tohoku region, the predicted wind power generation by the hybrid ensemble is relatively accurate (i.e., most of the observed wind power generation is included in the 95th percentile), especially for forecast day 2. The forecast skill decreases gradually, while the extent of the PDF increases with respect to the forecast length, which could be regarded as a convergence of the PDF toward the climatological PDF. The forecast uncertainty varies substantially from day to day after forecast day 3 . As for the longer range (forecast day 7), we find wide variations in the predictability/uncertainty, which is known as windows for "forecasts of opportunity" [37-40] in relation to planetary-scale teleconnections.
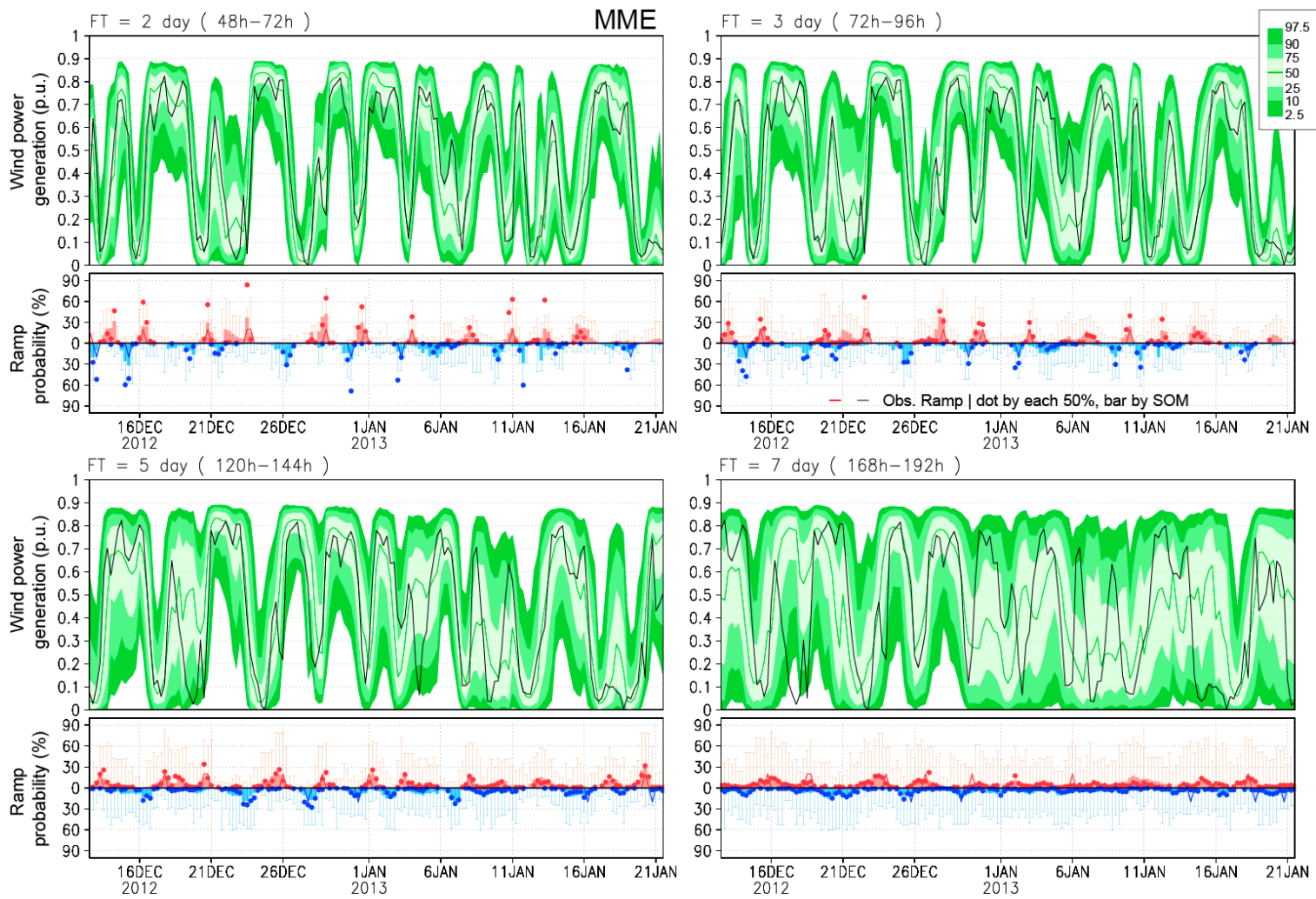

Figure 7. Forecasted 6-h wind power generation obtained from the hybrid ensemble for two-day, three-day, five-day, and seven-day forecasts around 1 January 2013. The black line represents the observed wind power generation. Forecasted PDFs of wind power generation obtained from the hybrid ensemble are represented by green shading. Each figure at the bottom shows the forecasted ramp probability obtained from the SOM nodes and the medium of each ensemble, as represented by red and blue bars and dots, respectively. Observed ramp-up and ramp-down events (actual state) are represented by the red and blue lines, respectively.

We have calculated the root mean square error (RMSE) for each ensemble forecast between the median of the probabilistic forecast and observations (Figure 8a) to measure the average forecast error. The ensemble forecast aims to construct the uncertainty information. As a metric of probabilistic 
forecast verification, we have also included the continuous ranked probability score (CRPS) [41], which is one of the measures of the integrated squared difference of the cumulative distribution function (CDF) of the forecasts (Figure 8b) from observations. The CRPS is commonly used to assess the respective accuracy of probabilistic forecasts. The RMSE and CRPS of the wind power forecasts are shown for the multi-model and each of the five ensemble forecasts for two to eight days' lead time. The results of the CRPS and RMSE are relatively similar to each other. Both the CRPS and RMSE show that the ECMWF ensemble forecasts have the best skill in the five models, while the UKMO has the second-best skill. The remaining models have a similar level of accuracy. The effect of combining the single-model systems can be seen in MME. The RMSE and CRPS of the MME result in lower RMSE and CRPS for all of the lead times.
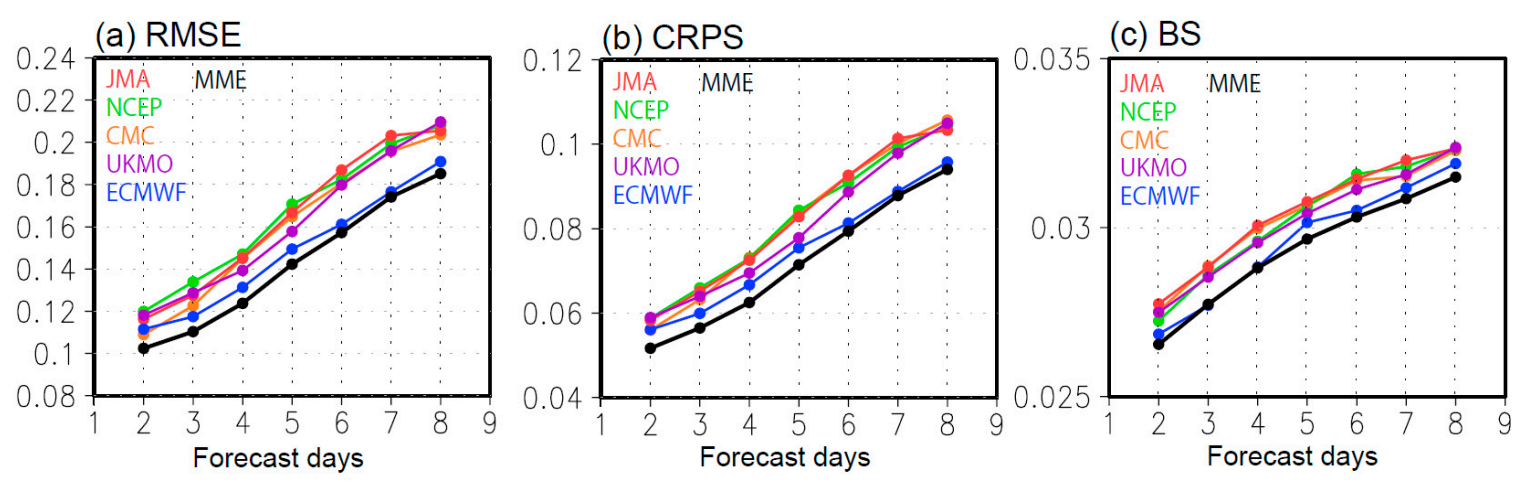

Figure 8. (a) Root mean square error (RMSE), (b) continuous ranked probability score, and (c) Brier score for wind power generation forecasts by CMC (orange), ECMWF (blue), JMA (red), NCEP (green), UKMO (purple), and multi-model ensembles (MME) (black) for the targeted region from April 2011 to May 2013.

In addition to the forecasts of wind power generation, we also assessed the forecast of wind ramp events provided from the hybrid ensemble forecasts in probabilistic form. Commonly used verification methods for probabilistic forecasts are Brier score (BS) and reliability diagrams. To check the skill of the wind power forecasts, we used BS (Figure 8c) and reliability diagrams obtained from the multi-center and single-center ensembles for FY2011/2012. Figure 9 presents the reliability diagrams for probabilistic forecasts of wind power ramps over the region for that period. The centers of circles in the upper-left and lower-right of the diagonal line indicate an underestimation and overestimation of the risks, respectively. Most of the single-center ensemble forecasts tend to overestimate the risks, even with a lead forecast time of two days. The MME forecasts are significantly more reliable than the other weather ensembles for most of the lead times. The BS shows that the ECMWF has the best, while the UKMO has the second-best performance.

From the BS and slopes of the reliability diagrams, the multi-model ensemble shows relatively good skill compared to the single-center ensemble forecasts for almost all of the lead times. The construction of multi-model ensembles can improve the reliability throughout the forecast periods. The MME plots are nearly diagonally at the lead times of two to four days, while showing improved forecast skill for the ramp events for all of the lead times compared with the single model.

The improvement in wind ramp forecasts using the multi-model ensemble can be attributed to the increase in ensemble spread. A particular single-center forecast cannot always show the best performance in predicting ramp events, since the ensemble forecasts are occasionally very different. If some forecasts show high occurrence probabilities of ramp events, the other single-center ensemble forecasts do not necessarily show similarly high probabilities. In this case, the MME will result in low probabilities of occurrence. However, if most of the models are in good agreement regarding the occurrence of ramp events, it can lead to the improvement of reliability with high forecast probabilities. 


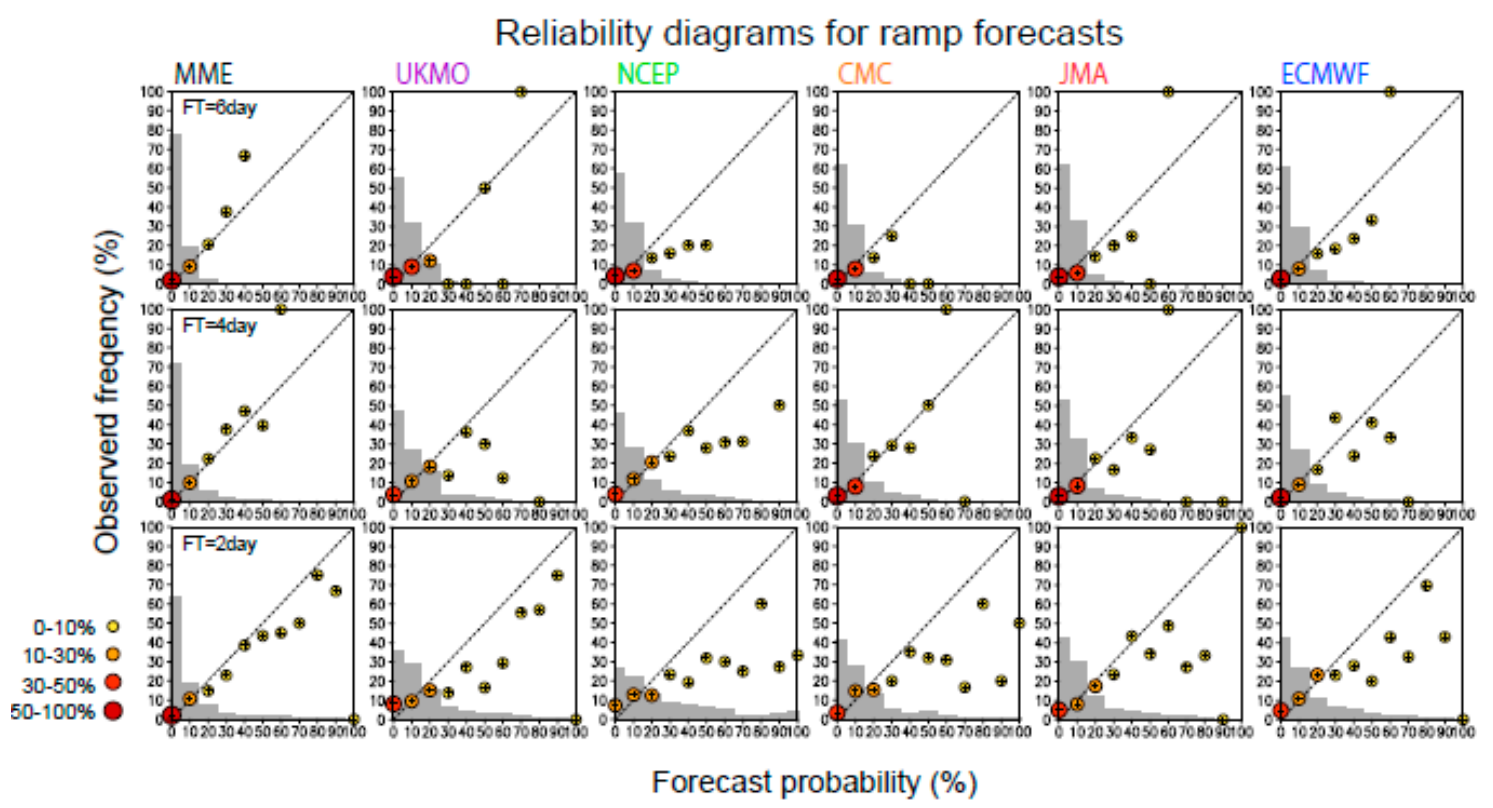

Figure 9. Reliability diagrams for two-day, four-day, and six-day forecasts of wind ramp events in the Tohoku region from April 2011 to May 2013 obtained from the MME and five single-center ensembles. Frequency histograms are represented by gray bars and dot colors.

In addition to the effect of the multi-model ensemble, we have also shown the effect of the analog ensemble. The results of the single-ensemble method for the JMA ensemble forecasts and single-control forecast are presented in Figure 10. To evaluate the skill of the hybrid ensemble forecasts, we have also shown the forecast skill obtained from the conventional (non-hybrid) scheme, namely, the nearest-neighbor single analog method. The single analog method was carried out for comparing the results obtained from only one single analog dataset (MME 1 analog; i.e., matching the pattern to the closest pattern in the reanalysis). Instead of the use of the SOM method, the MME 1 analog predicted by picking up the reconstructed wind power data corresponding with the highest similarity in the atmospheric variables among the reanalysis data. We used Euclidean distance to measure the similarity of the weather pattern and pick up one WP presenting the highest similarity. We compared the results obtained from the MME 1 analog for the JMA forecast and the full MME.

(a) RMSE

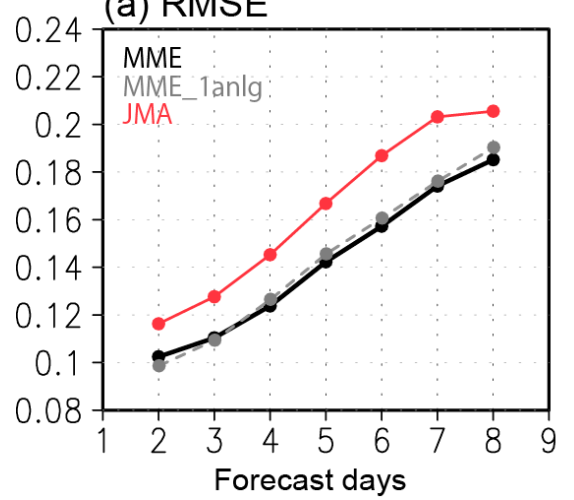

\section{(b) CRPS}

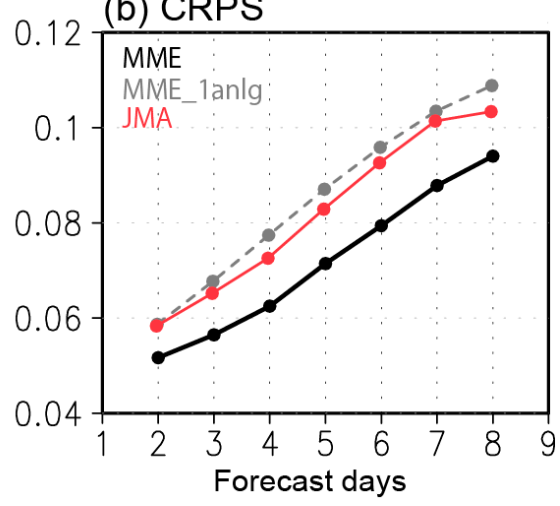

Figure 10. Same as Figure $8 \mathrm{a}, \mathrm{b}$, except for wind power generation forecasts, using a single analog method for MME (gray). JMA (red) and MME (black) are the same as in Figure 8.

The resulting RMSE increases with increasing forecast lead time for all of the approaches, as shown in Figure 10a. We see a relative improvement in RMSE when using the multi-model ensembles. Interestingly, the difference in the RMSE of the median between the SOM analog ensemble and the 
single ensemble is very small. This seems to be related to a decrease in the number of valid analogs near the outer edge of the weather attractor. Appropriate analog numbers are considered to be different from one time to another. Compared to the RMSE, the prediction accuracy tends to be better in the analog ensemble method in the CRPS (Figure 10b). Despite the decrease in computational cost, the SOM-based analog ensemble method improves the skill versus conventional methods. This change could be attributed to the effect of the analog ensemble, which makes a more realistically forecasted PDF by obtaining the nearby sample points.

\section{Discussion and Conclusions}

In addition to evaluating the wind power potential [42], forecasting the variability of wind power is one of most important challenges in the energy sector [2]. In this study, we present the application of a SOM-based analog ensemble method for medium-range wind power generation/variation forecasts by using multi-center ensemble forecast data, in order to support system operation for transmission grid operators. As discussed in a previous study of wind power and climate in Japan [19], the wind ramp events in East Japan are largely affected by synoptic circulation. Most of the ramp-up events in Japan are due to approaching extra-tropical cyclones, while most of the ramp-downs are due to the reduced gradients of surface pressure corresponding to the cover of anticyclonic highs. The complex relationships between synoptic-scale WPs and regionally integrated wind power generation were studied to obtain synoptic scale weather information around Japan by SOMs. We have shown the applications of the SOM not only for analyzing multi-model ensemble forecasts, but also for the probabilistic forecasting of wind power generation and ramp events. The skill of the wind power forecasts based on the hybrid multi-model analog ensemble method using SOM was evaluated for the Tohoku region in East Japan. From the results, we showed that regionally integrated wind power production and variability, in relation to synoptic-scale WPs, can be predicted days in advance. The medium-range wind power forecast over Japan is relatively skillful. The skill predictability of multi-center ensembles improves that of single-center ensembles significantly when using the perfect prognosis approach. We confirmed that the SOM-compressed analog ensemble improves the forecasts and can then be an effective estimation method when a very large number of ensemble members (i.e., multi-center ensemble) and historical local data are used. The information obtained about predictive uncertainty can be fruitful as an information source for decision-making in transmission grid operation.

The multi-model ensemble surpasses that of the single-model for both deterministic and probability forecasts [3]. As the multi-center ensemble cancels individual systematic errors in each model, it can provide more realistic estimates than individual forecast systems [43,44]. Since the hybrid ensemble method presented in this study needs SOM analysis in advance, it is a computationally expensive and complicated method. However, this method can be an alternative method when we cannot use long-term wind power data. The hybrid ensemble method that was presented in this study has the potential to provide more realistic PDFs. Moreover, it can be employed rapidly, with a very large number of ensemble forecasts outputs ranging from medium-range (weekly) to long-range (seasonal). While it cannot include various feedback processes at a local scale, our method is particularly advantageous from a computational cost perspective, as it quickly provides a first-order estimation of weather impacts on regional wind power resources.

Author Contributions: M.O. wrote the paper; M.O. and S.K. developed methodology; project administration, D.N.; funding acquisition, S.K.

Funding: This research was supported by "Research and development projects on power system output fluctuation-related technology" of the New Energy and Industrial Technology Development Organization (NEDO). Part of this work was also supported by the JSPS KAKENHI Grant Number JP 17K18426.

Acknowledgments: The authors express their gratitude to the Ogimoto Lab at the Institute of Industrial Science, the University of Tokyo for providing the observed area-integrated wind power generation data, derived from a collaborative research project between the lab and the Japan Wind Power Association (JWPA). 
Conflicts of Interest: The authors declare no conflict of interest.

\section{References}

1. World Energy Outlook: 2016. International Energy Agency. OECD/IEA: 2016. p. 684. Available online: https:/ / www.iea.org/media/publications/weo/WEO2016Chapter1.pdf (accessed on 20 September 2018).

2. Marquis, M.; Wilczak, J.; Ahlstrom, M.; Sharp, J.; Stern, A.; Smith, J.C.; Calvert, S. Forecasting the wind to reach significant penetration levels of wind energy. Bull. Am. Meteorol. Soc. 2011, 92, 1159-1171. [CrossRef]

3. Park, Y.Y.; Buizza, R.; Leutbecher, M. TIGGE: Preliminary results on comparing and combining ensembles. Q. J. R. Meteorol. Soc. 2008, 134, 2051-2066. [CrossRef]

4. Lorenz, E.N. Atmospheric predictability as revealed by naturally occurring analogs. J. Atmos. Sci. 1969, 26, 639-646. [CrossRef]

5. Zorita, E.; von Storch, H. The analog method as a simple statistical downscaling technique: Comparison with more complicated methods. J. Clim. 1999, 12, 2474-2489. [CrossRef]

6. Timbal, B.; McAvaney, B.J. An analogue-based method to downscale surface air temperature: Application for Australia. Clim. Dyn. 2001, 17, 947-963. [CrossRef]

7. Gutierrez, J.M.; Cofino, A.S.; Cano, R.; Sordo, C. Analysis and downscaling multi-model seasonal forecasts in Peru using self-organizing maps. Tellus A 2005, 57, 435-447. [CrossRef]

8. Garcia-Morales, M.B.; Dubus, L. Forecasting precipitation for hydroelectric power management: How to exploit GCM's seasonal ensemble forecasts. Int. J. Climatol. 2007, 27, 1691-1705. [CrossRef]

9. Delle Monache, L.; Eckel, T.; Rife, D.; Nagarajan, B. Probabilistic weather prediction with an analog ensemble. Mon. Weather Rev. 2013, 141, 3498-3516. [CrossRef]

10. Junk, C.; Delle Monache, L.; Alessandrini, S.; von Bremen, L.; Cervone, G. Predictor-weighting strategies for probabilistic wind power forecasting with an analog ensemble. Meteorol. Z. 2015, 24, 361-379. [CrossRef]

11. Davò, F.; Alessandrini, S.; Sperati, S.; Delle Monache, L.; Airoldi, D.; Vespucci, M.T. Post-processing techniques and principal component analysis for regional wind power and solar irradiance forecasting. Sol. Energy 2016, 134, 327-338. [CrossRef]

12. Kohonen, T. Self-organized formation of topologically correct feature maps. Biol. Cybern. 1982, 43, 59-69. [CrossRef]

13. Hewitson, B.C.; Crane, R.G. Consensus between GCM climate change projections with empirical downscaling: Precipitation downscaling over South Africa. Int. J. Climatol. 2006, 26, 1315-1337. [CrossRef]

14. Borah, N.; Sahai, A.K.; Chattopadhyay, R.; Joseph, S.; Abhilash, S.; Goswami, B.N. Self-organizing map-based ensemble forecast system for extended range prediction of active/break cycles of Indian summer monsoon. J. Geophys. Res. Atmos. 2013, 118, 1-13. [CrossRef]

15. Ohba, M.; Nohara, D.; Kadokura, S.; Toyoda, Y. Rainfall Downscaling of Weekly Ensemble Forecasts using Self-Organizing Maps. Tellus A 2016, 68, 29293. [CrossRef]

16. Eckel, F.A.; Delle Monache, L. A hybrid NWP-analog ensemble. Mon. Weather Rev. 2016, 144, 897-911. [CrossRef]

17. Kobayashi, S.; Ota, Y.; Harada, Y.; Ebita, A.; Moriya, M.; Onoda, H.; Onogi, K.; Kamahori, H.; Kobayashi, C.; Endo, H.; et al. The JRA-55 Reanalysis: General specifications and basic characteristics. J. Meteorol. Soc. Jpn. 2015, 93, 5-48. [CrossRef]

18. Harada, Y.; Kamahori, H.; Kobayashi, C.; Endo, H.; Kobayashi, S.; Ota, Y.; Onoda, H.; Onogi, K.; Miyaoka, K.; Takahashi, K. The JRA-55 Reanalysis: Representation of atmospheric circulation and climate variability. J. Meteorol. Soc. Jpn. 2016, 94, 269-302. [CrossRef]

19. Ohba, M.; Nohara, D.; Kadokura, S. Impacts of Synoptic Circulation Patterns on Wind Power Ramp Events in East Japan. Renew. Energy 2016, 96, 591-602. [CrossRef]

20. Ohba, M.; Kadokura, S.; Nohara, D.; Toyoda, Y. Anomalous Weather Patterns in Relation to Heavy Precipitation Events in Japan during the Baiu Season. J. Hydrometeorol. 2015, 16, 688-701. [CrossRef]

21. Reusch, D.B.; Alley, R.B.; Hewitson, B.C. North Atlantic climate variability from a self-organizing map perspective. J. Geophys. Res. 2007, 112, D02104. [CrossRef]

22. Ito, M.; Miyoshi, T.; Masuyama, H. The characteristics of the torus self-organizing map. In Proceedings of the 16th Fuzzy System Symposium Akita Japan Society for Fuzzy and Systems, Akita, Japan, 6-8 September 2000; pp. 373-374. 
23. Alessandrini, S.; Delle Monache, L.; Sperati, S.; Nissen, J. Short-term wind power forecasting with an analog ensemble. Renew. Energy 2015, 76, 768-781. [CrossRef]

24. Vanvyve, E.; Delle Monache, L.; Monaghan, A.J.; Pinto, J. Wind resource estimates with an analog ensemble approach. Renew. Energy 2015, 74, 761-773. [CrossRef]

25. Klink, K. Atmospheric circulation effects on wind speed variability at turbine height. J. Appl. Meteorol. Climatol. 2007, 46, 445-456. [CrossRef]

26. Cutler, N.J.; Outhred, H.R.; MacGill, I.F.; Kepert, J.D. Predicting and presenting plausible future scenarios of wind power production from numerical weather prediction systems: A qualitative ex ante evaluation for decision making. Wind Energy 2012, 15, 473-488. [CrossRef]

27. Hamlington, B.D.; Hamlington, P.E.; Collins, S.G.; Alexander, S.R.; Kim, K.Y. Effects of climate oscillations on wind resource variability in the United States. Geophys. Res. Lett. 2015, 42, 145-152. [CrossRef]

28. Gibson, P.B.; Cullen, N.J. Synoptic and sub-synoptic circulation effects on wind resource variability-A case study from a coastal terrain setting in New Zealand. Renew. Energy 2015, 78, 253-263. [CrossRef]

29. Pryor, S.C.; Schoof, J.; Barthelmie, R.J. The impact of non-stationarities in the climate system on the definition of a 'normal wind year': A case study from the Baltic. Int. J. Climatol. 2005, 25, 735-752. [CrossRef]

30. Davy, R.; Milton, J.; Russel, C.; Coppin, P. Statistical downscaling of wind variability from meteorological fields. Bound Layer Meteorol. 2010, 135, 165-175. [CrossRef]

31. Peña, J.C.; Aran, M.; Cunillera, J.; Amaro, J. Atmospheric circulation patterns associated with strong wind events in Catalonia. Nat. Hazards Earth Syst. Sci. 2011, 11, 145-155. [CrossRef]

32. Brayshaw, D.J.; Troccoli, A.; Fordham, R.; Methven, J. The impact of large scale atmospheric circulation patterns on wind power generation and its potential predictability: A case study over the UK. Renew. Energy 2011, 36, 2087-2096. [CrossRef]

33. Ely, C.R.; Brayshaw, D.J.; Methven, J.; Cox, J.; Pearce, P. Implications of the North Atlantic Oscillation for a UK-Norway renewable power system. Energy Policy 2013, 62, 1420-1427. [CrossRef]

34. Gibson, P.B.; Cullen, N.J. Regional variability in New Zealand's wind resource linked to synoptic-scale circulation: Implications for generation reliability. J. Appl. Meteorol. Climatol. 2015, 54, 944-958. [CrossRef]

35. Deppe, A.J.; Gallus, W.A.; Takle, E.S. A WRF ensemble for improved wind speed forecasts at turbine height. Weaher Forecast. 2013, 28, 212-228. [CrossRef]

36. Matsumoto, J. The seasonal changes in Asian and Australian monsoon regions. J. Meteorol. Soc. Jpn. 1992, 70, 257-273. [CrossRef]

37. Marshall, A.G.; Hudson, D.; Hendon, H.H.; Pook, M.J.; Alves, O.; Wheeler, M.C. Simulation and prediction of blocking in the Australian region and its influence on intra-seasonal rainfall in POAMA-2. Clim. Dyn. 2014, 42, 3271-3288. [CrossRef]

38. Hudson, D.; Alves, O.; Hendon, H.H.; Marshall, A.G. Bridging the Gap between Weather and Seasonal Forecasting: Intraseasonal Forecasting for Australia. Q. J. R. Meteorol. Soc. 2011, 137, 673-689. [CrossRef]

39. White, C.J.; Hudson, D.; Alves, O. ENSO, the IOD and the intraseasonal prediction of heat extremes across Australia using POAMA-2. Clim. Dyn. 2014, 43, 1791-1810. [CrossRef]

40. Johnson, N.C.; Collins, D.C.; Feldstein, S.B.; L'Heureux, M.L.; Riddle, E.E. Skillful wintertime North American temperature forecasts out to four weeks based on the state of ENSO and the MJO. Weather Forecast. 2014, 29, 23-38. [CrossRef]

41. Hersbach, H. Decomposition of the continuous ranked probability score for ensemble prediction systems. Weather Forecast. 2000, 15, 559-570. [CrossRef]

42. Archer, C.L.; Jacobson, M.Z. Evaluation of global wind power. J. Geophys. Res. Atmos. 2005, 110, D12110. [CrossRef]

43. Matsueda, M.; Tanaka, H.L. Can MCGE outperform the ECMWF ensemble? SOLA 2008, 4, 77-80. [CrossRef]

44. Johnson, C.; Swinbank, R. Medium-range multimodel ensemble combination and calibration. Q. J. $R$. Meteorol. Soc. 2009, 135, 777-794. [CrossRef]

(c) 2018 by the authors. Licensee MDPI, Basel, Switzerland. This article is an open access article distributed under the terms and conditions of the Creative Commons Attribution (CC BY) license (http:/ / creativecommons.org/licenses/by/4.0/). 